\title{
On the Applicability of Taylor's Theory to the Underwater Blast Response of Composite Plates
}

\author{
Y.P. Sone Oo ${ }^{\mathrm{a}, *}$, H. Le Sourne ${ }^{\mathrm{b}}$, O. Dorival ${ }^{\mathrm{c}}$ \\ ${ }^{a}$ GeM Institute (UMR CNRS 6183) - Calcul-Meca, Nantes, France. \\ ${ }^{b}$ GeM Institute (UMR CNRS 6183) - Icam Nantes campus, France. \\ ${ }^{c}$ Clément Ader Institute (FRE CNRS 3687) - Icam Toulouse and Nantes campuses, France.
}

\begin{abstract}
The response of a plane, air-backed plate attached to a rigid baffle when subjected to an underwater plane shock wave is investigated numerically and analytically. The numerical simulation takes into account a full three-dimensional fluid model involving water cavitation effects. Different numerical models are employed and validated by comparing against various experimental results from the literature. The validated numerical setup is then used to simulate the underwater shock response of simply-supported, air-backed, carbon-fiber/epoxy rectangular plates attached to a rigid baffle. Simplified analytical solution is developed based on two-step approach. The two stages considered are earlytime phase that adapts Taylor's fluid-structure theory to calculate an impulsive velocity for the plate and long-time phase that involves determining its free oscillation response within linear elastic domain, taking into account the water-added mass effect. Finally, the applicability of the proposed method is investigated by performing different numerical simulations regarding various combinations of peak pressures and decay times, change of aspect ratios, change of materials as well as ply orientations.
\end{abstract}

Keywords: Underwater explosion (UNDEX), composite rectangular plate, Taylor's theory, fluid-structure interaction (FSI), analytical, numerical

\footnotetext{
${ }^{*}$ Corresponding author.

Email address: ye-pyae.sone-oo@icam.fr (Y.P. Sone Oo)
} 


\section{Introduction}

Underwater explosions have long been the focus of naval research since World War II. Over the past decades, several advances have been brought forth by many researchers in the field. Consequently, a considerable body of literature already existed. References [1, 2, 3, provide a wide overview of many of those underwater explosion researches.

\subsection{Review of experimental work}

Experimental tests in the past were done in an underwater detonics basin using real explosives, see [4, 5, 6. Such works are indeed very important since they give ample understanding of the physical phenomena, accounting for threedimensional and other non-linear effects that could be expected in practice. However, the cost to conduct such experiments can be very high. Also, it is difficult to perform experiments in non-military settings and sometimes, data acquisition such as measuring spherical wave fronts and pressure signatures could be quite intricate [7. The attention has, therefore, been shifted on conducting underwater explosion (UNDEX) tests in a lab-scaled environment that employs a shock tube, see [7, 8, 9]. The benefits are that it becomes much easier to control the accuracy as well as the data measurement. In addition, a wide range of studies could be performed only within a limited budget although the size of the test apparatus might still impose limitations on the specimen sizes.

\subsection{Review of theoretical and numerical work}

Among the earliest theoretical works, Taylor's one-dimensional (1D) fluidstructure interaction (FSI) theory [10] is a well-known and a widely adapted approach due to its simplicity and effectiveness. The major finding is that the momentum transferred to the plate could be substantially reduced by decreasing

35 the plate areal mass or its acoustic impedance due to the FSI. It can be said that the early-time interaction effect is properly accounted for in the method. However, the late time response is not considered in Taylor's theory. Thus, Deshpande et al. 8], who had proved the FSI theory of Taylor by experiments, 
pointed out that a decoupled model based only on Taylor's free-standing plate theory might underestimate the structural response.

The theoretical studies performed by Kennard [11, 12] give much insights in regards to many physical aspects of the UNDEX problems as well as bulk cavitation that may occur in the vicinity of the immersed structure. Nearly 70 years later, Schiffer et al. [13] adapted the findings of Kennard [11] and proposed an analytical model for a 1D spring-supported, air-backed and water-backed rigid plates subjected to a plane shock wave, explicitly taking into account the cavitation of the fluid as well as the influence of the hydrostatic pressure. Another mathematical model that describes both the cavitation and wave propagation in bilinear fluid was given by Bleich and Sandler [14. In 1984, these results had been used by Felippa and DeRuntz [15] as a benchmark to validate their numerical method that could treat cavitation.

With the development of Doubly Asymptotic Approximations (DAAs) during the 1970s by Geers [16, 17, the paradigm for treating UNDEX problems has been shifted to a new era. DAAs have been implemented in Underwater Shock Analysis (USA) code and then incorporated into various commercial finite element tools such as LS-DYNA, NASTRAN, etc. [18. These are time domain differential equations that approach exactness at both high and low frequencies and a smooth transition in-between. The governing equations are expressed in terms of wet surface variables only and thus, it is not needed to explicitly model 60 the surrounding fluid. The disadvantage, as will be shown in this paper, is that DAA model alone is not able to correctly capture the initiation, development and collapse of cavitation bubbles.

Felippa and DeRuntz [15] have proposed a more rigorous numerical method for considering the cavitation in which the acoustic elements are coupled with ${ }_{65}$ DAA non-reflecting boundaries (NRB). Acoustic cavitating fluid volume elements, which use a cost-effective computational scheme involving displacement potential as the only primary unknown, have first been developed by Newton [19]. The combination of these elements with DAA solvers has certain advantages. For example, cavitation can be properly accounted for with the use of a 
bilinear fluid model and by limiting the vapor tensile pressures. There would also be no need to wait for the shock wave propagation since arbitrary reference time $(t=0)$ could be adjusted for any arbitrary location inside the fluid domain through the use of DAA NRB solvers.

Indeed, these numerical solutions involving DAA approach are very power75 ful but, as shown by Barras [20, they can be very time-consuming and demand much competence from the users. As a consequence, numerical approach is not well-suited for preliminary design stage when numerous loading scenarios as well as different structural configurations have to be tested. In this case, simplified analytical solutions become more relevant as they can not only provide rapid and reasonably accurate solutions but can also be used to validate numerical solutions for simple cases such as a plate or a cylinder, thus giving insights to the problems at hands. In this paper, a simplified analytical solution is developed to predict the response of simply-supported, air-backed composite rectangular plates subjected to a plane shock wave. Derivations are done by following the two-step impulse-based approach analogous to the work of Brochard et al. 21. The first stage deals with the calculation of an impulsive velocity for the plate using Taylor's FSI theory and the second stage with determination of its deformation, taking into account the water-added mass which can be associated with the reloading or deceleration of the immersed plate.

There have also been a few other researchers in the past who have attempted to use impulsive loading to idealize the underwater blast, for example [22, 23] for the metallic sandwich panel responses. More recently, Schiffer and Tagarielli 24] proposed an analytical model for the composite circular plates, taking into account of the FSI before and after the cavitation and using the position of the flexural waves to determine the zones of the cavitated fluid. However, considering explicitly the non-linearity of cavitation and propagation of flexural wave fronts has led to intricate non-linear differential equations. Hoo Fatt and Sirivolu 25] have also developed an analytical model by introducing Taylor's FSI method into Lagrange's equations of motions in order to analyze the air and water blast responses of the sandwich panels. Cavitation is considered by 
setting the total pressure to zero and by iterating in time step to find the location and time of the first cavitation. However, water-added mass or reloading caused by reattachment of the cavitated water is not considered, thus leading to underestimation of the result for both air-backed and water-backed UNDEX cases.

\subsection{Scope of the paper}

The objectives of the current paper are two-fold. First of all, in order to compare the developed analytical solutions, it is required a validated finite element setup that could consider both cavitation and the surrounding 3D effects. Two experimental data from the literature and one provided by DGA (Délégation Générale de l'Armement of the French Ministry of Defense) are used to validate the numerical solutions. Three different numerical approaches are considered in this paper to model the coupled FSI phenomena, namely, (1) Non-linear finite element explicit code LS-DYNA involving acoustic volume elements, (2) LS-DYNA/USA coupled with DAA boundary element solver, and (3) LS-DYNA/USA with DAA non-reflecting boundary element (NRB) solver coupled to fluid acoustic elements. Indeed, it is the intention of the authors to evaluate the performance and check the validity of different FE approaches and then select the most suitable setup for later use as a reference. Only then, the simplified analytical solutions are compared against the validated FE simulation results. Another purpose of this paper is to investigate the limitations of the proposed analytical approach. Finally, it is worth noting that to gain insights about the important underlying phenomena of the air-backed linear elastic plates, the non-linearity due to large deformation, structural damping, failure as well as hydrostatics effects are not considered in this paper.

\section{Theoretical background}

\subsection{Explosive loading}

Detonation of an explosive charge gives rise to not only a primary shock wave in a spherical form propagating away from the charge but also the pulsation of 
the gas bubble that could generate a secondary pressure pulse having 10-15\% of the peak pressure. At a sufficiently far stand-off distance, these spherical shock waves can be considered as plane and the influence of the bubble pulse could be ignored. According to Cole [1], the plane shock pressure wave can be approximated as:

$$
P(t)=P_{0} e^{-t / \tau}, \quad \text { for } 0 \leq t \leq \tau
$$

where $P_{0}$ is the peak pressure, $t$ is the time variable, and $\tau$ is the decay time defined as the time required for the peak pressure to fall to $1 / e$ of its peak value. According to the Principle of Similarity, these can be related to the charge mass $C$ and stand-off distance $R$ as:

$$
\begin{gathered}
P_{0}=K_{1}\left(\frac{C^{1 / 3}}{R}\right)^{A_{1}} \\
\tau=K_{2} C^{1 / 3}\left(\frac{C^{1 / 3}}{R}\right)^{A_{2}}
\end{gathered}
$$

where $K_{1}, A_{1}, K_{2}$, and $A_{2}$ are constants that depend on the type of the explosives used.

The findings of Kennard [12] has led to characterization of the different physical phenomena of a plate impinged by a plane shock pressure wave by four characteristic times, namely: decay time $(\tau)$ of the incident shock wave (Eq. 3), cavitation inception time $\left(\tau_{c}\right)$ - the time required for the shock wave to set the plate into motion at maximum velocity, diffraction time $\left(T_{d}\right)$ - the time required for the shock wave to propagate from the plate center to the edges, and swing time $\left(T_{s}\right)$ - the time required for the plate to reach its first peak of deflection. Using these four characteristic times, Kennard [12] was able to identify four different sequences of phenomena that could arise in an event of a shock wave impinging a plate or diaphragm. These cases can be summarized as follows:

140 (1) Case 1: Cavitation occurs in the plate vicinity and its effect prolongs until the plate has reached its peak deflection.

(2) Case 2: Cavitation occurs in the plate vicinity but collapses shortly after and increases the response of the plate as a reloading effect. 
(3) Case 3: Cavitation does not occur and the plate has relatively long swing time compared to the decay time of the loading.

(4) Case 4: Cavitation does not occur and the plate has relatively short swing time compared to the decay time of the loading.

These findings will be related to the different case studies investigated in this paper.

\section{Analytical model}

Consider a simply-supported rectangular composite plate having the sides $a$, $b$ and uniform thickness $h$ is submerged in an infinite fluid domain. A standard Cartesian coordinate $(x, y, z)$ system is defined at the origin and mid-surface of the plate as shown in Fig. 1. The displacements in the $x, y, z$ directions are denoted as $u, v$ and $w$ respectively. Each $k^{\text {th }}$ orthotropic ply is oritentated at an angle $\theta^{(k)}$ with respect to the $x$-axis. First-order shear deformation theory (FSDT) and Lagrangian Energy equation are considered to derive the mechanical model of the plate in the absence of water. The in-air mechanical model is then extended to include FSI effects by using two-step impulse-based approach which comprises Taylor's FSI theory for the early time and water-added mass for the late time. Hydrostatics pressure, structural damping and failure effects are not considered in this paper.

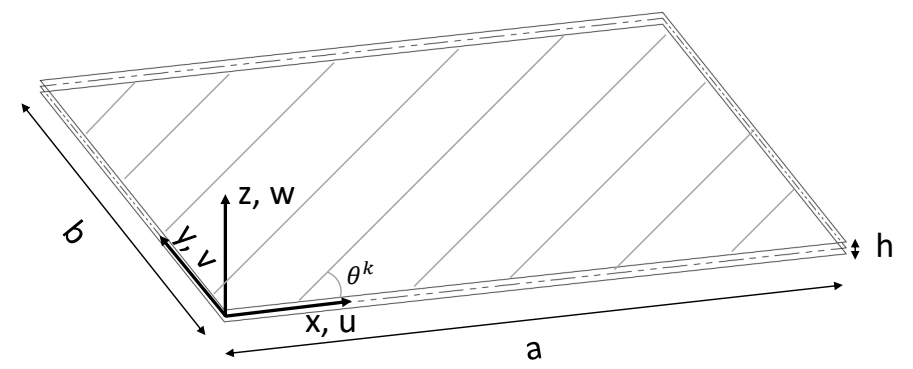

Figure 1: Panel geometry and coordinate system of the problem formulation 


\subsection{Non-immersed mechanical model}

According to FSDT, the transverse displacement is independent of the thick-

165

$\epsilon_{x z}$ and $\epsilon_{y z}$, are accounted for such that the transverse normals rotate with respect to the mid-surface after deformation. The in-plane displacements $|u|$ and $|v|$ are assumed negligibly small as compared with the transverse displacement $|w|$ so that the originally 5 Degree of Freedoms (DOFs) problem is reduced to only 3 DOFs.

The linear strain-displacement relations are written as follows:

$$
\begin{aligned}
& \epsilon_{x x}=z \frac{\partial \psi_{x}}{\partial x}, \quad \epsilon_{y y}=z \frac{\partial \psi_{y}}{\partial y}, \quad \gamma_{x y}=z\left(\frac{\partial \psi_{x}}{\partial y}+\frac{\partial \psi_{y}}{\partial x}\right) \\
& \gamma_{x z}=\frac{\partial w}{\partial x}+\psi_{x}, \quad \gamma_{y z}=\frac{\partial w}{\partial y}+\psi_{y}, \quad \gamma_{z z}=0
\end{aligned}
$$

To satisfy the simply-supported boundary conditions for the rectangular plate, a modal based-approach is adopted. Displacement is obtained as a superposition of simply-supported normal modes with the following double summations:

$$
\begin{aligned}
w(x, y, t) & =\sum_{m=1}^{\infty} \sum_{n=1}^{\infty} W_{m n} \sin \left(\frac{m \pi x}{a}\right) \sin \left(\frac{n \pi y}{b}\right) \\
\psi_{x}(x, y, t) & =\sum_{m=1}^{\infty} \sum_{n=1}^{\infty} \Psi_{x_{m n}} \cos \left(\frac{m \pi x}{a}\right) \sin \left(\frac{n \pi y}{b}\right) \\
\psi_{y}(x, y, t) & =\sum_{m=1}^{\infty} \sum_{n=1}^{\infty} \Psi_{y_{m n}} \sin \left(\frac{m \pi x}{a}\right) \cos \left(\frac{n \pi y}{b}\right)
\end{aligned}
$$

where $W_{m n}, \Psi_{x_{m n}}$ and $\Psi_{y_{m n}}$ are three generalized coordinates, $m$ and $n$ are mode numbers in $x$ - and $y$-directions respectively.

The lamina constitutive relations are described using 2D plane stress assumption. For any $k^{\text {th }}$ layer of the orthotropic lamina with an arbitrary orientation $\theta^{(k)}$, the stress-strain relationship can be written as:

$$
\left\{\begin{array}{c}
\sigma_{x x} \\
\sigma_{y y} \\
\sigma_{x y}
\end{array}\right\}^{(k)}=\left[\begin{array}{lll}
\bar{Q}_{11} & \bar{Q}_{12} & \bar{Q}_{16} \\
\bar{Q}_{12} & \bar{Q}_{22} & \bar{Q}_{26} \\
\bar{Q}_{16} & \bar{Q}_{26} & \bar{Q}_{66}
\end{array}\right]^{(k)}\left\{\begin{array}{c}
\epsilon_{x x} \\
\epsilon_{y y} \\
\gamma_{x y}
\end{array}\right\}
$$


and for transverse shear as:

$$
\left\{\begin{array}{l}
\sigma_{y z} \\
\sigma_{x z}
\end{array}\right\}^{(k)}=\left[\begin{array}{ll}
\bar{Q}_{44} & \bar{Q}_{45} \\
\bar{Q}_{45} & \bar{Q}_{55}
\end{array}\right]^{(k)}\left\{\begin{array}{l}
\gamma_{y z} \\
\gamma_{x z}
\end{array}\right\}
$$

where $\bar{Q}_{i j}^{(k)}$ from Eq. 6 and 7 is the reduced transformed stiffness matrix based on engineering constants. The detailed formulations to obtain $\bar{Q}_{i j}^{(k)}$ for normal 175 and shear components can be found in any classical composite textbooks (e.g., see Reddy [26]).

By using lamina constitutive relations (Eq. 6 and 7) and then by integrating the corresponding stresses with respect to the thickness, the well-known relationships for force and moment resultant to strains can be obtained:

$$
\left\{\begin{array}{l}
\{N\} \\
\{M\}
\end{array}\right\}=\left[\begin{array}{ll}
{[A]} & {[B]} \\
{[B]} & {[D]}
\end{array}\right]\left\{\begin{array}{l}
\left\{\epsilon^{0}\right\} \\
\{\kappa\}
\end{array}\right\}, \quad\left\{\begin{array}{l}
Q_{y} \\
Q_{x}
\end{array}\right\}=K_{s}\left[\begin{array}{ll}
A_{44} & A_{45} \\
A_{45} & A_{55}
\end{array}\right]\left\{\begin{array}{l}
\gamma_{y z} \\
\gamma_{x z}
\end{array}\right\}
$$

where $\{N\}$ and $\{M\}$ are column vectors of in-plane and bending moment resultants respectively, $\left\{\epsilon^{0}\right\}$ is the membrane strain matrix, $\{\kappa\}$ is the curvature matrix, $K_{s}$ is the shear correction factor to account for the non-uniformity and parabolic shape of the shear stress distribution throughout the thickness, $[A]$, $[B]$, and $[D]$ are extensional, bending-extensional coupling and bending stiffness matrices and $\left(A_{44}, A_{45}, A_{55}\right)$ are shear stiffnesses respectively. These values can be obtained using $\bar{Q}_{i j}^{(k)}$ and the $z$ coordinates of the plies as:

$$
\begin{array}{rr}
A_{i j}=\sum_{k=1}^{N} \bar{Q}_{i j}^{(k)}\left(z_{k+1}-z_{k}\right), & B_{i j}=\frac{1}{2} \sum_{k=1}^{N} \bar{Q}_{i j}^{(k)}\left(z_{k+1}^{2}-z_{k}^{2}\right) \\
D_{i j}=\frac{1}{3} \sum_{k=1}^{N} \bar{Q}_{i j}^{(k)}\left(z_{k+1}^{3}-z_{k}^{3}\right), & A_{o p}^{s}=\sum_{k=1}^{N} \bar{Q}_{o p}^{(k)}\left(z_{k+1}-z_{k}\right)
\end{array}
$$

where $A_{o p}^{s}=\left(A_{44}, A_{45}, A_{55}\right)$ are the shear stiffnesses (op $\left.=44,45,55\right)$.

By substituting Eq. 5- 9, the following expressions for kinetic energy $T$ and the strain energy $U$ can be derived:

$$
T=\frac{a b}{8} \sum_{m=1}^{\infty} \sum_{n=1}^{\infty}\left[I_{1} \dot{W}_{m n}^{2}+I_{2}\left(\dot{\Psi}_{x_{m n}}^{2}+\dot{\Psi}_{y_{m n}}^{2}\right)\right]
$$




$$
\begin{aligned}
U=\frac{a b}{8} \sum_{m=1}^{\infty} & \sum_{n=1}^{\infty}\left[D_{11}\left(\frac{m \pi}{a}\right)^{2} \Psi_{x_{m n}}^{2}+2 D_{12}\left(\frac{m n \pi^{2}}{a b}\right) \Psi_{x_{m n}} \Psi_{y_{m n}}\right. \\
+ & D_{22}\left(\frac{n \pi}{b}\right)^{2} \Psi_{y_{m n}}^{2}+D_{66}\left(\frac{\pi}{a b}\right)^{2}\left(a n \Psi_{x_{m n}}+b m \Psi_{y_{m n}}\right)^{2} \\
& \left.+A_{44}\left(\frac{b \Psi_{y_{m n}}+\pi n W_{m n}}{b}\right)^{2}+A_{55}\left(\frac{a \Psi_{x_{m n}}+\pi m W_{m n}}{a}\right)^{2}\right]
\end{aligned}
$$

where $I_{1}$ and $I_{2}$ are mass and rotatory inertia whose corresponding expressions can be found using:

$$
I_{1}=\rho \sum_{k=1}^{N}\left(h_{k}-h_{k-1}\right), \quad I_{2}=\frac{1}{3} \rho \sum_{k=1}^{N}\left(h_{k}^{3}-h_{k-1}^{3}\right)
$$

where $\rho$ is the density of the material (be it orthotropic or isotropic), and $h_{k}$ is the thickness of each lamina.

Lagrangian energy approach previously used by other researchers such as [27, 25] is employed here to derive the governing equations for the plate. According to Lagrangian equation:

$$
\frac{d}{d t}\left(\frac{\partial T}{\partial \dot{q}_{l}}\right)+\frac{\partial U}{\partial q_{l}}=Q_{n c_{l}}
$$

where $T$ and $U$ are the kinetic energy (Eq. 10) and strain energy (Eq. 11), $Q_{n c_{l}}$ is the non-conservative external force, $q$ is the generalized coordinates, $\dot{q}$ is the rate of change of generalized coordinates with respect to time and $l$ is the number of degrees of freedom. In the present analysis, this number is three $\left(w, \psi_{x}, \psi_{y}\right)$ and since only initial impulsive velocity is considered, $Q_{n c_{l}}$ can be taken as zero.

When the two energy expressions, Eq. 10 and 11, are substituted in Eq. 13 , equations of motion can be derived. Since the eigen modes involved in Eq. 5 are orthogonal, the resulting equations of motion are uncoupled to each mode. These can be given in the matrix form as follows:

$$
\left[\begin{array}{ccc}
M_{1} & 0 & 0 \\
0 & M_{2} & 0 \\
0 & 0 & M_{3}
\end{array}\right]\left[\begin{array}{l}
\ddot{W}_{m n} \\
\ddot{\Psi}_{x_{m n}} \\
\ddot{\Psi}_{y_{m n}}
\end{array}\right]+\left[\begin{array}{ccc}
K_{11} & K_{12} & K_{13} \\
K_{12} & K_{22} & K_{23} \\
K_{13} & K_{23} & K_{33}
\end{array}\right]\left[\begin{array}{l}
W_{m n} \\
\Psi_{x_{m n}} \\
\Psi_{y_{m n}}
\end{array}\right]=\left[\begin{array}{l}
0 \\
0 \\
0
\end{array}\right]
$$


where the formulations to calculate $M_{1}, M_{2}, M_{3}, K_{i j}(i, j=1,2,3)$ are given in the Appendix

By neglecting the effect of rotatory inertia, that is $I_{2} \approx 0, M_{2}$ and $M_{3}$ become zero. Therefore, the three sets of equations can be reduced in just one ordinary differential equation with $W_{m n}$ as the only unknown as:

$$
M_{1} \ddot{W}_{m n}(t)+K_{m n} W_{m n}(t)=0
$$

with the initial conditions: $W_{m n}(0)=0$ and $\dot{W}_{m n}(0)=2 A_{m n} v_{i}$, where $K_{m n}$ is the stiffness (see Appendix), $v_{i}$ is the impulsive velocity whose corresponding formula will be provided in Eq. 19 of Subsection 3.2.1 and $A_{m n}=8 /\left(m n \pi^{2}\right)$ is the term related to mode shape $(m, n)$. Note that structural damping effect $C$ is assumed negligible in Eq. 15. Due to the symmetry of the loading and boundary conditions, only odd number terms $m, n=1,3,5$, etc. contribute to the plate response. The Eq. 15 is a classical free-vibration equation and its solution can simply be given as:

$$
W_{m n}=\frac{2 A_{m n} v_{i}}{\omega_{0_{m n}}} \sin \left(\omega_{0_{m n}} t\right)
$$

where $\omega_{0_{m n}}=\sqrt{\frac{K_{m n}}{M_{1}}}=2 \pi f_{0_{m n}}$ is the angular natural frequency for each mode shape $m$ and $n$.

\subsection{Analytical FSI model}

The in-air mechanical model developed in the previous section is extended to include the FSI effect when fluid is present on one side of the plate and air on the other side. A plane shock pressure wave is applied uniformly to the entire plate on the fluid side. Following the approach of Brochard et al. 21, the interaction between fluid and structure is divided into two phases. In the first phase, the impulsive velocity transmitted to the plate is determined by using Taylor's 1D FSI theory [10]. The deformation of the plate in this phase is assumed negligibly small and cavitation is supposed to occur in the neighboring of the plate at the end of this phase. In the second phase, the deformation is supposed to begin and the cavitation zone to collapse with no time gap. This 
collapse would launch an additional pressure, namely water-added inertia effect, onto the plate. This would increase the transverse deflection of the plate as well as reduce its natural frequencies, causing longer periods of oscillation compared to the in-air response.

Following the approach of Taylor [10, total pressure applied to the plate during the early-time phase is obtained by a linear superposition of incident, reflected and radiated pressures as follows:

$$
P(t)=2 P_{0} e^{-t / \tau}-\rho_{w} c_{w} \dot{W}
$$

where $\rho_{w}$ and $c_{w}$ are water density and speed of sound in water respectively. The factor ' 2 ' in the former term comes from doubling of the pressure as if the plate is rigid and perfectly reflective upon arrival of the shock wave. The latter term $-\rho_{w} c_{w} \dot{W}$ represents a rarefaction term, which would decrease or even diminish the double pressure due to the plate movement. When the total pressure falls below the vapor pressure, cavitation would occur either on the surface of the plate or inside the fluid domain. In the case of Taylor's theory, it is assumed that cavitation always occur all over the surface of the plate. By solving the following equation of motion:

$$
\ddot{W}=\frac{2}{\rho h} P_{0} e^{-t / \tau}-\frac{\rho_{w} c_{w} \dot{W}}{\rho h}
$$

with the initial conditions $W(0)=0$ and $\dot{W}(0)=0$, Taylor has proposed the following analytical solutions for the free rigid 1D plate:

$$
\begin{gathered}
v_{i}=\frac{2 P_{0} \tau}{\rho h} \psi^{-\frac{\psi}{\psi-1}} \\
\tau_{c}=\frac{\tau \ln \psi}{\psi-1}
\end{gathered}
$$

where $v_{i}$ is the maximum impulsive velocity, $\tau_{c}$ is the cavitation inception time when $P(t)$ becomes zero and maximum $v_{i}$ is reached. $\psi=\frac{\rho_{w} c_{w} \tau}{\rho h}$ is the FSI coefficient relating decay time and areal mass $\rho h$ of the plate, whose significance 
will be highlighted later in Subsection 5.2 where various analytical results are evaluated. The reduced transferred impulse due to the movement of the plate can be calculated using:

$$
I_{t}=2 I_{0} \psi^{-\frac{\psi}{\psi-1}}
$$

where $I_{0}=P_{0} \tau$ is the applied impulse related to incident wave.

Note that only the maximum impulsive velocity (Eq. 19p and cavitation inception time (Eq. 20) of the Taylor's FSI theory [10 have been expressed in this paper. For the rest of the formulations regarding displacement, acceleration, pressure and so on, the reader is referred to the original paper of Taylor [10]. When Eq. 19 is applied in Eq. 16, closed-form analytical solution entitled only impulsive velocity for a $2 \mathrm{D}$ composite plate is obtained. This, however, still does not take into account the water-added mass effect that should appear at some time after $t>\tau_{c}$. This is discussed in the subsequent subsection.

\subsubsection{Long-time phase}

Deformation is supposed to begin only when the plate reaches its maximum impulsive velocity. It is considered here that the kinetic energy acquired by the plate during the first phase will be transferred to the deformation energy. In addition, during the plate deceleration phase, additional pressure due to 220 water inertial effect is assumed to act onto the plate, following the collapse of cavitation.

For a rectangular plate vibrating in water, the water-added mass per area can be determined by using Greenspon (1961) 28 formulation as:

$$
M_{a_{m n}}=\frac{1}{2} \rho_{w} b f(a / b) A_{m n}^{2}
$$

where $M_{a_{m n}}$ is the added mass of water, $f(a / b)=1.5(a / b)^{3}-3.12(a / b)^{2}+$ $2.6(a / b)+0.0098$ is the correction term for different aspect ratios of the plate $(0 \leq f(a / b) \leq 1)$ for $a \leq b$, and $A_{m n}=8 /\left(m n \pi^{2}\right)$ is a correction term for simply-supported boundary conditions and odd number modes $(m, n=$ $1,3,5, \ldots)$. This added-mass formulation of Greenspon, Eq. 22 . consists of some approximations on the mode shape term by assuming the entire plate as 
a rectangular piston with a deflection equal to the average of the mode shape. Thus, the above formulation is accurate only for the first mode. The final (wet) natural frequencies now become:

$$
\omega_{m n}=\sqrt{\frac{K_{m n}}{M_{1}+M_{a_{m n}}}}
$$

The equation of motion of the immersed air-backed plate related to the odd number mode $(m, n)$ is:

$$
\left(M_{1}+M_{a_{m n}}\right) \ddot{W}_{m n}(t)+K_{m n} W_{m n}(t)=0
$$

The solution provided by Eq. 16 from in-air case can be used for water blast case too except that the angular natural frequency of water (Eq. 23) and Taylor's impulsive velocity (Eq. 19 should be considered for FSI response. As a consequence of having the extra mass, the natural frequencies of the plate will decrease and the periods of oscillation will get longer compared to the non-immersed case. The maximum swing time $T_{s}$ of the plate including the water-added mass can be approximated using fundamental mode as:

$$
T_{s} \approx \frac{\pi}{2 \omega_{11}}=T_{0} \sqrt{1+\frac{M_{a_{11}}}{M_{1}}}
$$

where $T_{0} \approx 1 /\left(4 f_{0_{11}}\right)$ is the approximate in-air swing time when the plate reaches its first peak of deflection.

\section{Comparison of different numerical approaches}

Four different numerical models, which will be presented in the following subsection, have been set up and confronted to the following experiments: (1) a circular steel plate tested in a detonics basin by Goranson and reported by Cole [1], (2) a circular glass-fiber plate tested using a shock tube in a lab-scaled environment and reported by Schiffer and Tagarielli [9], and (3) a circular steel plate tested by DGA in an underwater detonics basin and presented in the subsection 4.2 .3 of the present paper. The primary goal here is to have an idea about the dynamic behavior of both steel and composite plates while setting 
up a reliable numerical model to be used later as a reference to validate the proposed analytical model.

235 4.1. Details of the FE models

Typical finite element models related to four different numerical approaches are illustrated in Fig. 22. In all of the FE approaches, the plate model is constructed depending on the materials used in the experiment as follows:

- For circular steel plate model, MAT_PLASTIC_KINEMATIC with Belyschko-Tsay shell element formulation, five through-thickness integration points and a shear factor of 5/6 are applied. The plate has 35 and 24 elements in the radial and circumferential directions respectively. Strain rate is taken into account by using Cowper Symonds formulation in which the values $C=40$ and $p=5$ (for mild steel) are chosen.

- For circular composite (GRP) plate model, MAT_COMPOSITE_ DAMAGE with fully-integrated shell element, one integration point per ply are applied. Transverse shear correction is done by activating laminated shell theory. The GRP plate is meshed to have 14 and 10 elements in the radial and circumferential directions respectively.

Note that only a quarter of the model is required due to problem symmetry and the upward or downward direction of the loading does not matter.

\subsubsection{LS-DYNA (impulsive velocity)}

Fig. 2a shows a quarter plate model of LS-DYNA which is subjected to the initial impulsive velocity calculated by Taylor's FSI theory (Eq. 19). Thus, fluid is not modeled in this approach. Simply-supported boundary is considered on the outer plate edges and the symmetric boundary conditions are applied on the inner plate edges as shown.

\subsubsection{LS-DYNA (only acoustic)}

In this model shown in Fig. 2b, a pressure loading is prescribed on one end of the acoustic fluid column while the shell plate model with a 

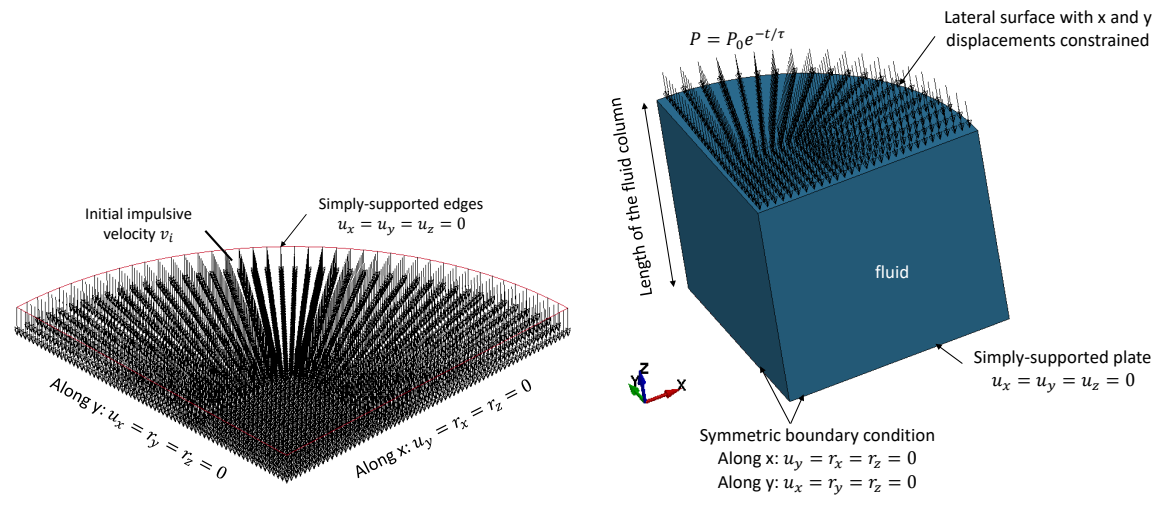

(a) LS-DYNA (only impulsive velocity)

(b) LS-DYNA (only acoustic)

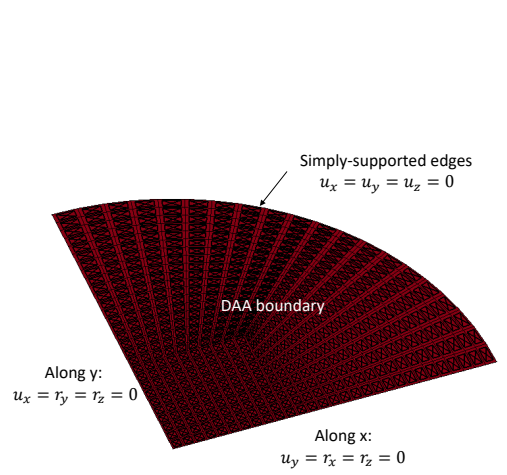

(c) LS-DYNA/USA (DAA2)

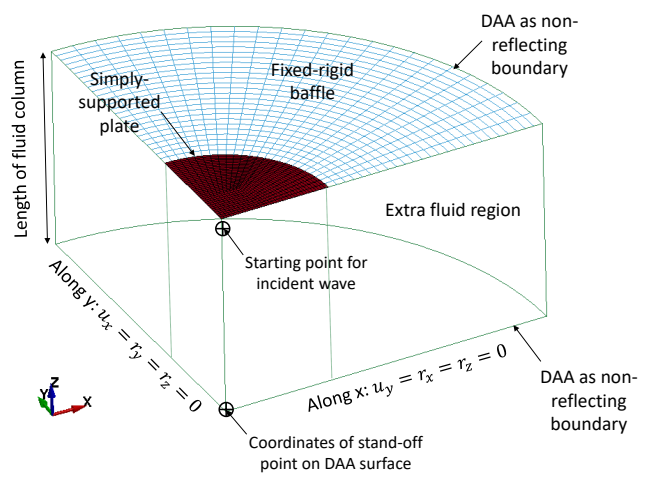

(d) LS-DYNA/USA + acoustics

Figure 2: Typical finite element models for the simulation of UNDEX using different numerical approaches: (a) LS-DYNA with only impulsive velocity (no fluid) model, (b) LS-DYNA with only acoustic elements model, (c) LS-DYNA/USA with DAA 2 boundary elements (no fluid) model, (d) LS-DYNA/USA acoustics coupled to DAA non-reflecting boundary model

simply-supported boundary condition is attached on the other end. BOUNDARY_ACOUSTIC_IMPEDANCE is applied on the same segment where the loading is applied so that the returning waves propagate out of the fluid domain and do not come back. A water column length of $0.25 \mathrm{~m}$ has been used. The author also tested a longer water-column length of $0.5 \mathrm{~m}$ but the results are similar to the ones that will be shown here. Acoustic solid element formulation in conjunction with MAT_ACOUSTIC is employed for the fluid model. Cavitation flag is turned on and vapor pressure is limited at zero. Applying the 
acoustic element formulation (ELFORM $=8$ or 14 ) in LS-DYNA requires the fluid element mesh to meet the following stability criteria [29]:

$$
\frac{\rho_{w} D}{\rho h}<2.5
$$

where $\rho_{w}$ and $D$ are the density and the thickness of the acoustic elements adjacent to the structural element whereas $\rho$ and $h$ are density and thickness of the structural shell element respectively. In accordance with this criterion, the thickness of the fluid mesh of $1 \mathrm{~mm}$ is used. Numerical damping of 0.25 is applied for stability reason too. As can be seen in Fig. 2b, the fluid meshes in $x-y$ plane are modeled the same as the structural meshes. At the fluid-structure interface, the nodes of the structure and the fluid are merged so that FSI is automatically treated. Symmetric boundary condition is applied for the inner fluid nodes and $x-y$ translations are constrained on the lateral fluid nodes.

\subsection{3. $L S-D Y N A / U S A\left(D A A_{2}\right)$}

A wet segment set is defined on the shell plate model to couple with secondorder Doubly Asymptotic Approximations $\left(\mathrm{DAA}_{2}\right)$ boundary elements, see Fig. 2c. A stand-off distance, desired peak pressure as well as the corresponding decay time can be defined through USA input card. Cavitation is treated only approximately in $\mathrm{DAA}_{2}$ model by limiting the total pressure at zero whenever its value becomes negative. Notice the difference in the assumption of surrounding boundary conditions on the lateral (outer) fluid faces between LSDYNA/USA (DAA ${ }_{2}$ ) model and LS-DYNA (only acoustic) model because LSDYNA (only acoustic) model employs a water domain of a finite extent whereas LS-DYNA/USA $\left(\mathrm{DAA}_{2}\right)$ model considers a plate immersed in an infinite or semi-infinite fluid domain in its formulation.

\subsubsection{LS-DYNA/USA acoustics}

As can be seen in Fig. 2d, the plate is attached to both the fixed rigid plate (shell element model with MAT_RIGID) and the acoustic fluid elements. The mesh of the plate is the same as the previous numerical models while that of the 
rigid baffle has about twice the mesh of the plate in the $x-y$ plane. The extra

The four FE models discussed above can be summarized as in Table 1

Table 1: Summary of four FE models simulated

\begin{tabular}{lcll}
\hline FE model & Fluid & Cavitation & Assumptions \\
\hline $\begin{array}{l}\text { LS-DYNA (only } \\
\text { impulsive velocity) }\end{array}$ & No & No & $\begin{array}{l}\text { No water inertia } \\
\text { or cavitation }\end{array}$ \\
$\begin{array}{l}\text { LS-DYNA } \\
\text { (only acoustic) }\end{array}$ & Yes & Yes & Finite extent of water \\
LS-DYNA/USA (DAA $)$ & No & Yes & $\begin{array}{l}\text { Infinite or semi-infinite } \\
\text { fluid domain }\end{array}$ \\
LS-DYNA/USA (acoustic) & Yes & Yes & $\begin{array}{l}\text { Semi-infinite fluid and } \\
\text { rigid baffle plate }\end{array}$ \\
\hline
\end{tabular}




\subsection{Comparisons and analyses}

4.2.1. A circular steel plate subjected to a plane shock wave (Goranson's test)

Experiment conducted by Goranson and reported by Cole [1] involves steel diaphragms that have different thicknesses and strengths. These are securely fastened to the equivalent of a heavy steel ring that has about $300 \mathrm{~mm}$ width and is mounted on the front of a heavy watertight structure. According to Kennard [12], this ring can be regarded as roughly equivalent to an infinite baffle. Charges of $0.45 \mathrm{~kg}$ TNT are employed to attack the test diaphragms from different stand-off distances. One of those tests is selected to use in our current study since it represents the response of a relatively thin plate subjected to a short decay loading. The parameters of explosive charge, the plate as well as material characteristics from the test are given in Tables 2 and 3 To compare with the experimental loading condition, a double decay formulation from [30] is applied here. It has the same profile as Cole's exponential formulation until $t<\tau$, but has a more accurate profile for the longer time upto $t=7 \tau$ written as:

$$
P_{i}(t)=\left\{\begin{array}{l}
0, \quad t<0 \\
P_{0} e^{-t / \tau}, \quad 0 \leq t<\tau \\
P_{0}\left(0.8251 e^{-1.338 t / \tau}+0.1749 e^{-0.1805 t / \tau}\right), \quad \tau \leq t \leq 7 \tau
\end{array}\right.
$$

The results of central-deflection versus time for different numerical models are plotted in Fig. 3. First of all, it can quickly be seen that the best match to the experimental result is that of LS-DYNA/USA acoustic model. Increase of the peak deflection around $1.7 \mathrm{~ms}$ is found to be due to the reloading associated

Table 2: Parameters of the explosive charge (Goranson's experiment [1])

\begin{tabular}{cccccc}
\hline$\rho_{w}\left(\mathrm{~kg} \cdot \mathrm{m}^{-3}\right)$ & $c_{w}\left(\mathrm{~m} \cdot \mathrm{s}^{-1}\right)$ & $C(\mathrm{~kg})$ & $R(\mathrm{~m})$ & $P_{0}(\mathrm{MPa})$ & $\tau(\mathrm{ms})$ \\
\hline 1025 & 1500 & 0.45 & 1.827 & 18.73 & 0.081 \\
\hline
\end{tabular}


Table 3: Characteristics of the plate and material used (Goranson's experiment [1])

\begin{tabular}{cccccc}
\hline$a(\mathrm{~m})$ & $h(\mathrm{~mm})$ & $\rho\left(\mathrm{kg} . \mathrm{m}^{-3}\right)$ & $E(\mathrm{GPa})$ & $\nu$ & $\sigma_{Y}(\mathrm{MPa})$ \\
\hline 0.2664 & 2.79 & 7800 & 204 & 0.3 & 240 \\
\hline
\end{tabular}

to the collapse of cavitation. Almost all of the FE models perform analogously to the in-air response upto $1 \mathrm{~ms}$. Significant differences are observed only after that time. Taylor's impulsive velocity result underestimates by about $30 \%$, a relative error with respect to the experimental value, when final deflections are compared. The final deflection obtained from LS-DYNA (only acoustic) approach is quite similar to the impulsive loading result, however, the behavior is totally different. Plastic straining near the central portion is found in both models (i.e., only acoustic and impulsive velocity) but the amount of straining in LS-DYNA (only acoustic) is even less than that of impulsive velocity simulation. The real reason is believed to be that when the fluid is modeled only up to a finite extent (Fig. 2b) together with the restraints in $x-y$ translations, the acoustic fluid contains the pressure and then seems to hinder the rebounding of the plate, making it unable to oscillate or causing a very slow return. The same behavior is observed in the composite plate model too, that will be discussed in the next section. The plate deflection post-processed from LS-DYNA/USA $\left(\mathrm{DAA}_{2}\right)$ model is overestimated by about $40 \%$ (a relative error from the experiment) since this model could not accurately capture the non-linear nature of the cavitation according to [15].

The result extracted from LS-DYNA/USA acoustic simulation can be related to the case 2 (cavitation with water reloading effect) of Kennard's finding. The values for cavitation inception time and the diffraction time for Goranson's plate model are obtained as $\tau_{c}=0.03 \mathrm{~ms}$ and $T_{d}=0.18 \mathrm{~ms}$ respectively. Cole [1] has mentioned that the occurrence of cavitation can be quickly checked by comparing $\tau_{c}$ and $T_{d}$. If $\tau_{c}<T_{d}$, this means cavitation would occur which happens to be the present case study. To observe the occurrence of cavita- 


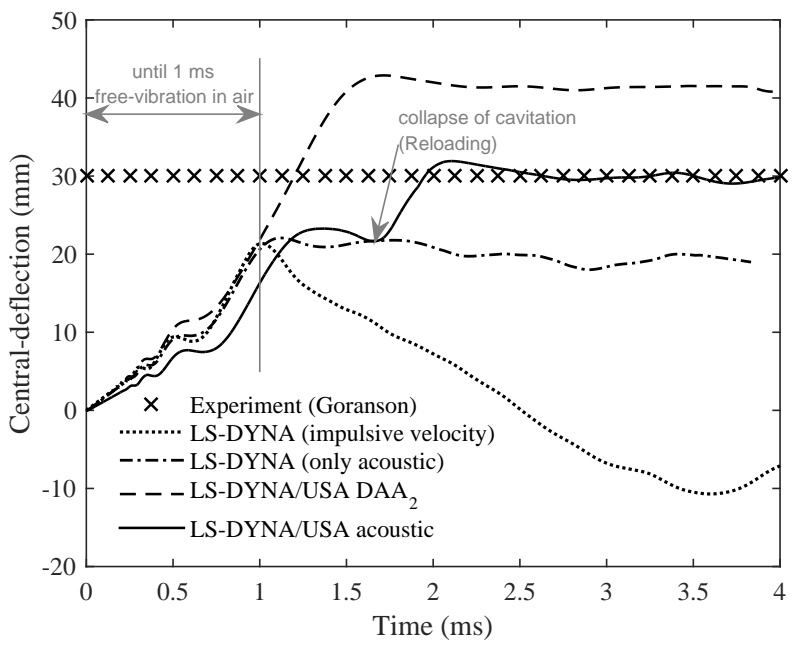

Figure 3: Comparison between central-deflection-time history results calculated by different numerical codes and Goranson's experimental result performed on steel circular plate

tion, pressure contours at various time steps are retrieved from LS-DYNA/USA acoustic simulations, see Fig. 4. The range of the pressure values is set at 0 - $10 \mathrm{MPa}$ and the region around the plate is zoomed for clear visibility. Soon after the arrival of the shock wave, cavitation arises quite rapidly due to the flexibility of the plate when subjected to a plane shock wave with a relatively short duration (Fig. 4a). It can be seen that the observation matches with the predicted theoretical value of cavitation inception time given by Eq. 20. According to [11, 13, the occurrence of cavitation could give rise to two breaking fronts that will propagate outward from the point of first cavitation. However, in the present case, only one breaking front can be seen since the other one occurs very close to or directly on the fluid-structure interface. Depending on the pressure and particle velocity in the fluid immediately around the breaking fronts, these fronts could arrest and remain stationary or reverse their directions and become closing fronts. At about the diffraction time shown in Fig. $4 \mathrm{~b}$, the breaking fronts propagating away from the bulk cavitation zone (blue color) can be seen. Since the traveling of these fronts are 3D in nature, the incoming water 


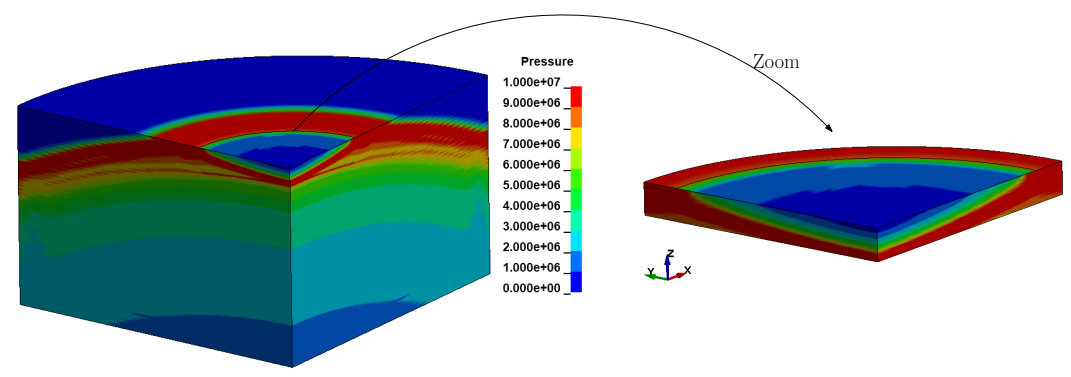

(a) At $t=\tau_{c}=0.03 \mathrm{~ms}$ (cavitation inception time)

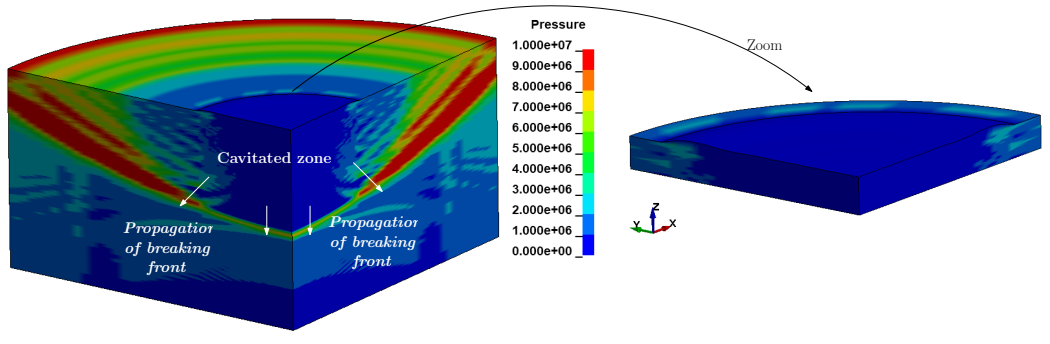

(b) At $t=T_{d}=0.18 \mathrm{~ms}$ (diffraction time)

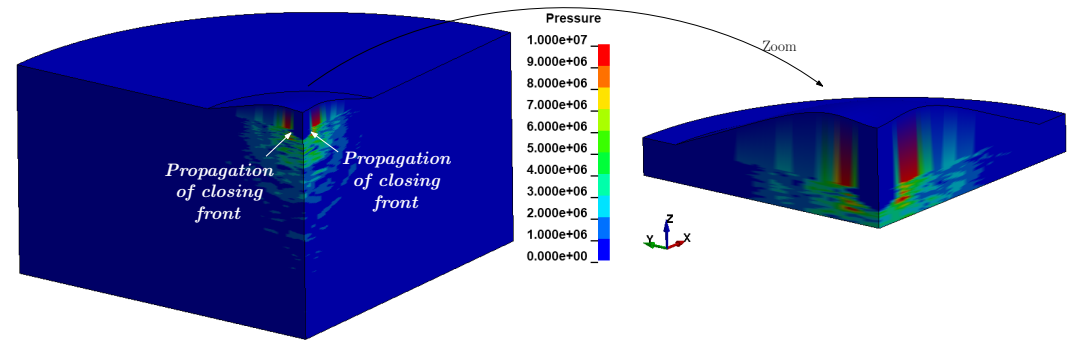

(c) At $t=1.7 \mathrm{~ms}$ (reloading time)

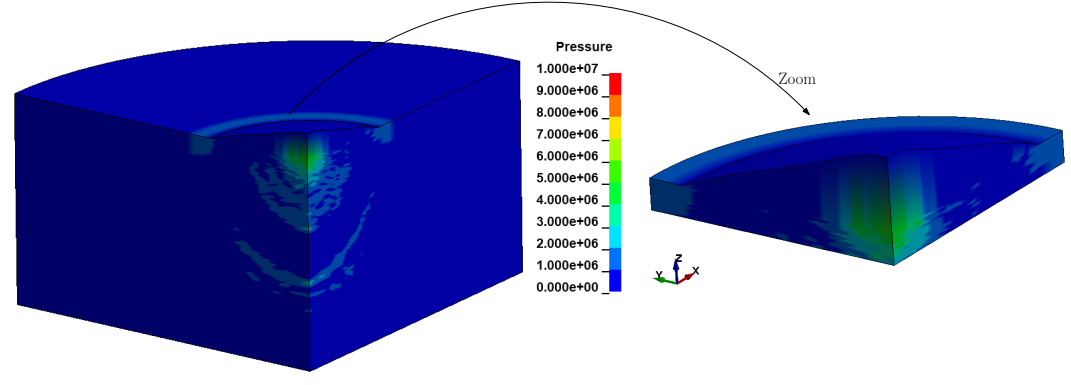

(d) At $t=2.1 \mathrm{~ms}$ (at the time of peak deflection)

Figure 4: Pressure contours at various time steps for LS-DYNA/USA acoustics model of Goranson's experiment (Plate deflection is amplified by 3 times for clear visibility) 
diffraction effect is almost blocked out and the plate oscillates analogously to an in-air response until $1 \mathrm{~ms}$. The water reloading then starts at about $1.7 \mathrm{~ms}$, see Fig. 4c. Indeed, such phenomenon can be associated to the collapse of the bulk cavitation due to the arrest of the breaking front and the return of closing fronts. Water reloading effect could generate an additional pressure wave, which further increases the final plate deflection by about 30\%, see Fig. 4d. Without this effect, the result of LS-DYNA/USA acoustics would be approximately the same as that of LS-DYNA (only acoustic) simulation. Thus, this case study clearly highlights the importance of $3 \mathrm{D}$ surrounding conditions as well as the possible effects of cavitation onto the structure.

Effect of the size of the rigid baffle. The authors also studied the effect of the rigid baffle by performing LS-DYNA/USA acoustics simulations using different baffle sizes. It was found out that result (with no rigid baffle) is approximately the same as that of LS-DYNA (only acoustic). Increasing the lateral dimension of the rigid baffle increases the plate deflection but this effect is bound by the inverse of the radius of the rigid baffle in accordance with Kirchhoff Retarded Potential Formulation [31. In other words, using larger and larger baffle sizes, the plate deflection will converge towards a certain value (30 $\mathrm{mm}$ in our case).

\subsubsection{A circular GRP plate subjected to a plane shock wave}

Schiffer and Tagarielli [9] have conducted their experiments in a laboratory environment using quasi-isotropic glass/vinylester and a woven carbon/epoxy plates. The test employs a transparent water-filled shock tube having a length of $2 \mathrm{~m}$ and diameter of $25 \mathrm{~mm}$. The composite specimens are supported by a clamping ring. A total of 14 test cases were reported in [9] in which different peak pressures and decay times were investigated. The dynamic plate deflections were recorded using a high-speed camera. In this paper, the experiment from 375 [9] that involves GRP plate ( $\left.a=12.5 \mathrm{~mm}, h=0.85 \mathrm{~mm}, \rho=1550 \mathrm{~kg} \cdot \mathrm{m}^{-3}\right)$ with a stacking sequence of $[0,45,90,-45]$ is selected for the comparison. The authors also checked other three cases from [9] and all of them correlate quite well to the numerical results. So, only one of these cases is evaluated in detail 
in this paper. The loading conditions as well as characteristics of the plate are given in Tables 4 and 5 respectively. The incident loading has an exponential profile as in Eq. 1. The length of the water column is modeled as $2 \mathrm{~m}$. Most of the FE model setups are the same as presented in Subsection 4.1. Note that LS-DYNA/USA acoustics model for Schiffer and Tagarielli's experiment does not comprise of the extra fluid so as to resemble the test tube setting of the experiment.

Central deflections obtained from various numerical models are plotted as a function of time in Fig. 5a Pressure-time history is retrieved from LSDYNA/USA acoustics model as shown in Fig. 5b/in order to have better insights about the results. First of all, it can be seen that the results post-processed from LS-DYNA (only acoustic) and LS-DYNA/USA acoustic models are very similar and correlate quite well with the experiment with a relative discrepancy of about $7 \%$ to the experimental maximum deflection. It should also be noticed that acoustic volume elements contained the pressure near the plate, which nonphysically prevents the elastic return of this latter. Expectation of such behavior (slow rebounding) in practice is a question since the trend of the experimental time-history result does not seem to show any of this behavior.

Similarly to the Goranson's case study, the results of LS-DYNA/USA $\left(\mathrm{DAA}_{2}\right)$ model overestimates the plate deflection (with a relative error of about $36 \%$ from the experiment) since such approach considers a plate immersed in an infinite

Table 4: Parameters of the loading and fluid (Experiment of Schiffer and Tagarielli 9])

\begin{tabular}{cccc}
\hline$\rho_{w}\left(\mathrm{~kg} \cdot \mathrm{m}^{-3}\right)$ & $c_{w}\left(\mathrm{~m} \cdot \mathrm{s}^{-1}\right)$ & $P_{0}(\mathrm{MPa})$ & $\tau(\mathrm{ms})$ \\
\hline 1000 & 1055 & 9 & 0.12 \\
\hline
\end{tabular}

Table 5: Characteristics of the circular GRP plate (Experiment of Schiffer and Tagarielli [9])

\begin{tabular}{ccccc}
\hline$E_{1}(\mathrm{GPa})$ & $E_{2}=E_{3}(\mathrm{GPa})$ & $\nu_{12}=\nu_{23}$ & $G_{12}=G_{13}(\mathrm{GPa})$ & $G_{23}(\mathrm{GPa})$ \\
\hline 27.8 & 5.0 & 0.3 & 1.86 & 1.92 \\
\hline
\end{tabular}



interface much later than the expected theoretical value. 


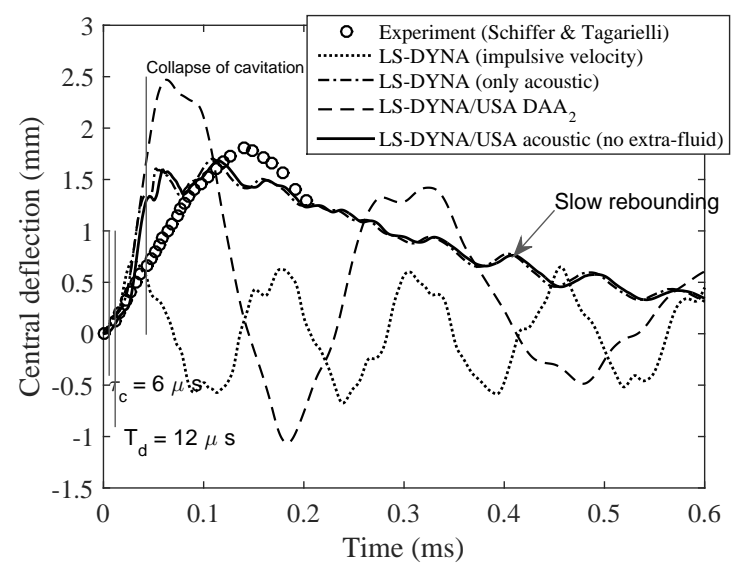

(a) Deflection Vs time

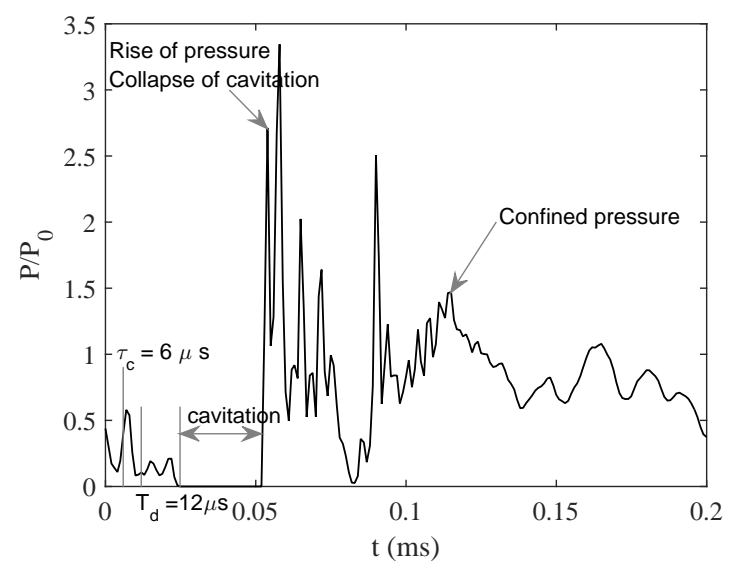

(b) $P(t) / P_{0}$ Vs time

Figure 5: Comparison of the numerical results with the experimental result of [9] conducted on circular GRP plate: (a) plot of central deflections obtained from different numerical approaches and experiment is given as a function of time, (b) normalized pressure $P / P_{0}$ obtained from LS-DYNA/USA (acoustics) simulation is plotted as a function of time

\subsubsection{A circular steel plate subjected to a plane shock wave (DGA test)}

An in-house test data provided by DGA is used to further test the validity of the LS-DYNA/USA acoustics approach. The test configurations are shown in Fig. 6 in which a steel circular plate of diameter $410 \mathrm{~mm}$ and thickness 4 $\mathrm{mm}$ is bolted to a watertight submerged frame. A TNT equivalent charge of 
$55 \mathrm{~g}$ is then detonated at $0.9 \mathrm{~m}$ stand-off distance, giving $P_{0}=18.86 \mathrm{MPa}$ and $\tau=0.04 \mathrm{~ms}$ with an exponential profile. The steel plate has Young modulus $(E=210 \mathrm{GPa})$, poisson ratio $(\nu=0.3)$, yield stress $\left(\sigma_{Y}=250 \mathrm{MPa}\right)$ and tangent modulus of $1680 \mathrm{MPa}$. The attached rigid plate is regarded as a semiinfinite baffle plate. The conditions for FE models are the same as presented in Subsection 4.1. The length and lateral dimensions of the fluid model in the LS-DYNA/USA acoustic approach are defined as two times the radius of the target plate.

Time histories of the plate are shown in Fig. 6c. It can be observed that the numerical result agrees quite favorably with the experiment. Note that the experimental central-deflection is measured with the help of a laser before the first pulsation of the gas bubble. Knowing that the time associated with the bubble first contraction is at least 100 orders of magnitude greater than the decay time of the primary shock wave, only the time history upto $3 \mathrm{~ms}$ is shown. The numerical result is oscillating between $4 \mathrm{~mm}$ and $4.6 \mathrm{~mm}$, and compared to the experimental value (about $4 \mathrm{~mm}$ ), the maximum relative error is about $15 \%$ (at about $2.7 \mathrm{~ms}$ ). Such discrepancy is probably due to the fact that the simply-supported boundary condition used in the FE model is not the exact representation of the test which may be somewhere between simply-supported and clamped conditions.

According to the studies performed in Subsections 4.2.1, 4.2.2 and 4.2.3, it can be said that LS-DYNA/USA acoustic model has the best correlations with the experiment. LS-DYNA with only impulsive velocity given by Taylor's theory could lead to significant underestimations especially for thick plates, which oscillate in high frequencies. Using LS-DYNA $\left(\mathrm{DAA}_{2}\right)$ approach (without surrounding fluid model) could overestimate the responses since it could not capture cavitation correctly. Using LS-DYNA/USA (only acoustics) simulations with only finite extent of water may lead to underestimations and an unnaturally slow rebounding of the plate due to confined pressures in the neighboring acoustic volume elements. Therefore, LS-DYNA/USA (acoustic) model setup will be used as reference for further comparisons with the analytical results. 


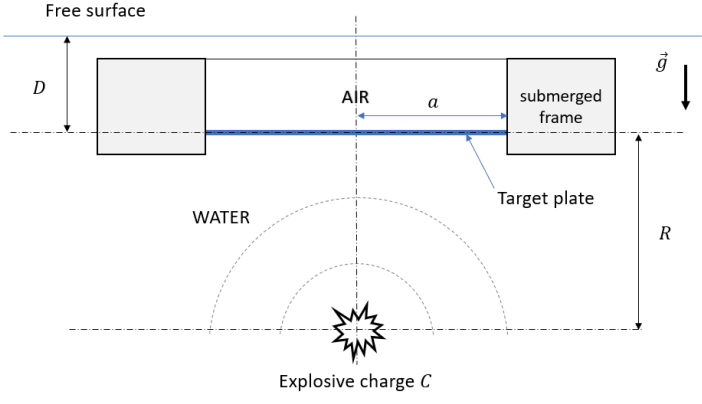

(a) Test configuration

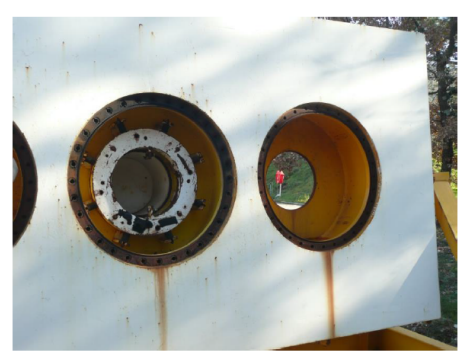

(b) Submerged frame (Front view)

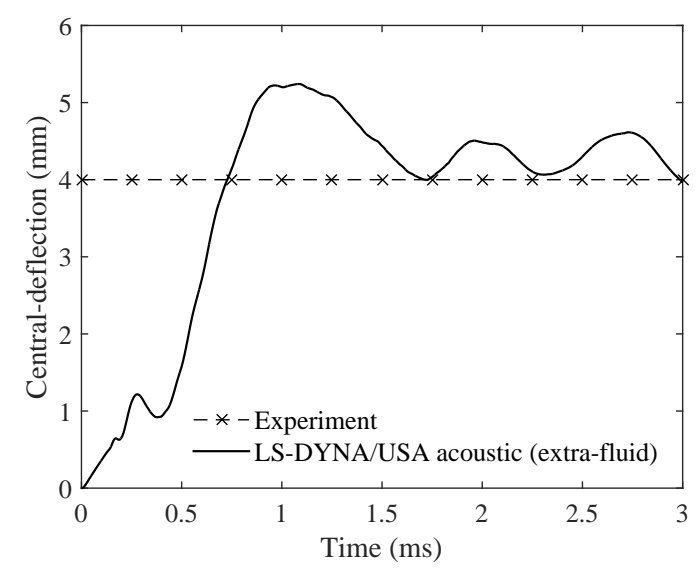

(c) Central-deflection Vs time

Figure 6: DGA test setup performed on a circular steel plate subjected to a TNT equivalent charge of $55 \mathrm{~g}$ and comparison of the central-deflections with LS-DYNA/USA acoustic simulation

\section{Case studies using analytical models}

465

\subsection{Materials and load cases}

Carbon-fiber/epoxy (CFRP) laminates having a uniform thickness of $5.76(\mathrm{~mm})$, density of $1548\left(\mathrm{~kg} \cdot \mathrm{m}^{-3}\right)$, and the stacking sequence of $[ \pm 45 / 0 / 0 / 0 / \pm 45 / 0 / 0 / 0 / 90 / 90]_{S}$ is used to demonstrate the analytical solutions. The length of the edges $(a=b)$ will be varied to have aspect ratios of $a / h=69.4$ (thin plate) and $a / h=17.4$ (thick plate). The material characteristics, Table 6, are taken from quasi-static tests performed by the authors. 
Comparisons are first performed for in-air (non-immersed) plate model by applying a range of impulsive velocity loading. Then, investigations regarding FSI will be performed as shown in Table 7 in which the cases denoted with C-1a to $\mathrm{C}-1 \mathrm{~d}$ represent thin CFRP plate and $\mathrm{C}-2 \mathrm{a}$ to $\mathrm{C}-2 \mathrm{c}$ for the thick CFRP plate. The peak pressures and decay times are selected so as to give the same transferred impulse $I_{t}$ given by Taylor's theory (Eq. 21). The obtained results are then compared with the results of LS-DYNA/USA acoustic simulations including rigid baffle plate and the extra fluid model.

\subsection{Results and discussions}

\subsubsection{Impulsive loading (non-immersed case)}

In Fig. 7, the numerical results regarding the peak central deflections are found within $\pm 10 \%$ of the analytical results except for the large aspect ratio (or large deflection). Since geometric non-linearity related to large displacement is not considered, the analytical formula is accurate only upto about one time the thickness of the plate $(W / h \approx 1)$. Note that the obtained analytical solutions consider the first five odd number modes $(m, n=1,3, \ldots, 9)$ where a convergence of the solution is found. Since the energy dissipation is more or less the same for a given applied impulse $I_{0}$, the deformation energy agrees much better than those of the central-deflections for all aspect ratios (Fig. 7b). In other words, when the plate deflection exceeds its thickness, some of the kinetic energy is absorbed by an additional stretching mode caused by moderately large rotations, see Reddy [32] for more further details.

Table 6: Characteristics of the material (carbon-fiber/epoxy laminate)

\begin{tabular}{cccccc}
\hline $\begin{array}{c}E_{11} \\
(\mathrm{GPa})\end{array}$ & $\begin{array}{c}E_{22}=E_{33} \\
(\mathrm{GPa})\end{array}$ & $\begin{array}{c}\nu_{12}=\nu_{13} \\
-\end{array}$ & $\begin{array}{c}\nu_{23} \\
-\end{array}$ & $\begin{array}{c}G_{12}=G_{13} \\
(\mathrm{GPa})\end{array}$ & $\begin{array}{c}G_{23} \\
(\mathrm{GPa})\end{array}$ \\
\hline 138 & 8.98 & 0.281 & 0.385 & 3.66 & 3.24 \\
\hline
\end{tabular}




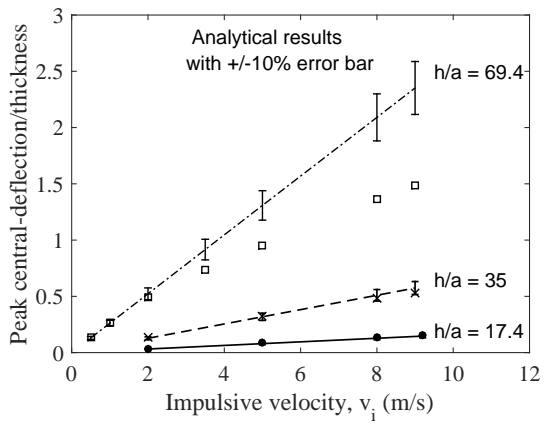

(a) Impulsive velocity Vs $w_{\max } / h$

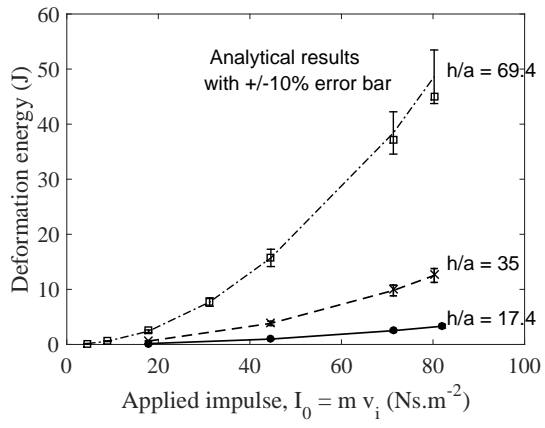

(b) Applied impulse Vs deformation energy

Figure 7: Comparison of in-air plate response subjected to the impulsive loading (Numerical results are shown with $\bullet, \times, \square$ and the analytical ones are shown with lines)

Table 7: Load cases for FSI studies

\begin{tabular}{|c|c|c|c|c|c|c|c|c|}
\hline $\begin{array}{l}\text { Cases } \\
(-)\end{array}$ & $\begin{array}{l}a \\
(\mathrm{~mm})\end{array}$ & $\begin{array}{l}h \\
(\mathrm{~mm})\end{array}$ & $\begin{array}{l}a / h \\
(-)\end{array}$ & $\begin{array}{l}f_{0} \\
(\mathrm{~Hz})\end{array}$ & $\begin{array}{l}P_{0} \\
(\mathrm{Mpa})\end{array}$ & $\begin{array}{l}\tau \\
(\mathrm{ms})\end{array}$ & $\begin{array}{l}I_{0} \\
\left(\mathrm{Nsm}^{-2}\right)\end{array}$ & $\begin{array}{l}I_{t} \\
\left(\mathrm{Nsm}^{-2}\right)\end{array}$ \\
\hline C-1a & 400 & 5.76 & 69.4 & 190 & 0.647 & 0.051 & 33 & 5.66 \\
\hline $\mathrm{C}-1 \mathrm{~b}$ & 400 & 5.76 & 69.4 & 190 & 0.551 & 0.167 & 92 & 5.66 \\
\hline C-1c & 400 & 5.76 & 69.4 & 190 & 0.524 & 0.335 & 175.5 & 5.66 \\
\hline C-1d & 400 & 5.76 & 69.4 & 190 & 0.509 & 0.67 & 341 & 5.66 \\
\hline $\mathrm{C}-2 \mathrm{a}$ & 100 & 5.76 & 17.4 & 2808 & 2.3 & 0.024 & 55.2 & 16.97 \\
\hline $\mathrm{C}-2 \mathrm{~b}$ & 100 & 5.76 & 17.4 & 2808 & 1.939 & 0.05 & 97 & 16.97 \\
\hline C-2c & 100 & 5.76 & 17.4 & 2808 & 1.632 & 0.192 & 313.3 & 16.97 \\
\hline
\end{tabular}

\subsubsection{Underwater explosive loading}

The investigations regarding the UNDEX loading including the FSI effect are performed on the 7 load cases specified in Table 7 by using LS-DYNA/USA acoustic approach as well as analytically. In order to generalize our study, the following dimensionless parameters are introduced:

$$
\begin{aligned}
\bar{W}_{\max } & =\frac{\rho_{w} c_{w}}{2 P_{0} \tau} W_{\max }, \quad \bar{v}_{i}=\frac{\rho_{w} c_{w}}{P_{0}} v_{i} \\
\psi & =\frac{\rho_{w} c_{w} \tau}{\rho h}, \quad \bar{I}=\frac{I_{t}}{I_{0}}=2 \psi^{\frac{\psi}{1-\psi}}
\end{aligned}
$$


of the water with the plate. On the other hand, when the load has relatively long decay time, the impulse-based nature of the current analytical approach could not capture well the continuing FSI phenomenon caused by the long loading duration. Two peaks can be seen in LS-DYNA/USA acoustics central deflection 


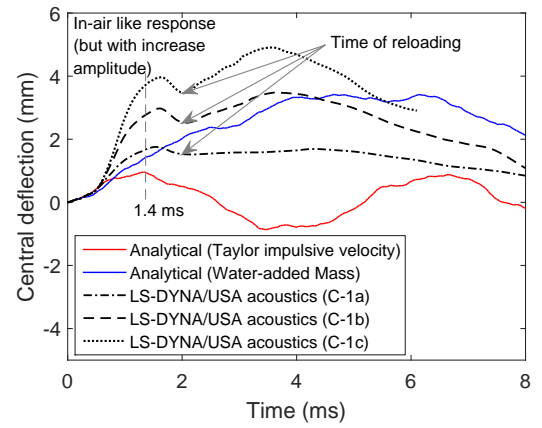

(a) Cases C1-a to c: Thin plate $(a / h=69.4)$

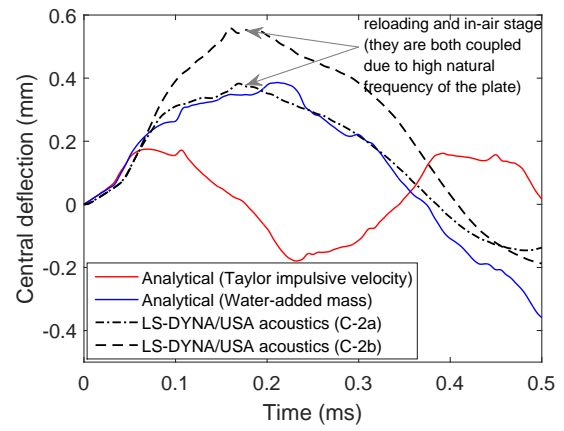

(b) Cases C2-a to b: Thick plate $(a / h=17.4)$

Figure 8: Comparison of central-deflection time histories between analytical and numerical methods for thin and thick rectangular CFRP plates

Sensitivity to peak pressure. Knowing that the proposed analytical formulations work well only for a certain range of decay times, the sensitivity due to the change in the peak pressures is investigated while keeping the same decay time. 


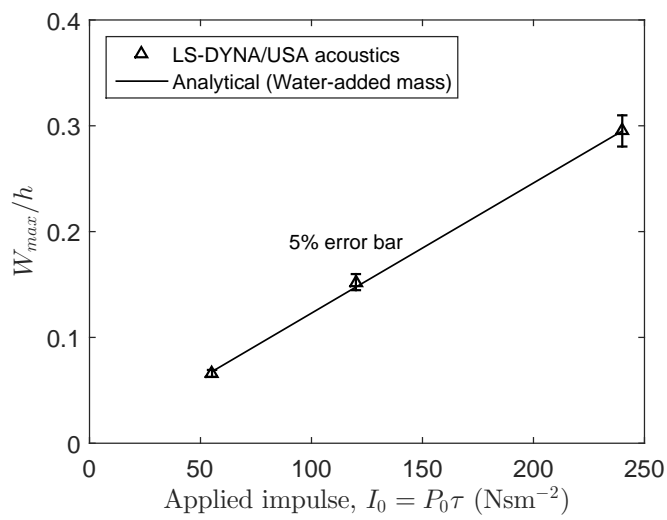

Figure 9: Results using same decay time $\tau$ but with different peak pressures $P_{0}(a / h=17.4)$

Decay time from case study C-2a, $\tau=0.024 \mathrm{~ms}$, is selected and results for different peak pressures are plotted in Fig. 9. As expected, the results show linear relationship as long as the maximum deflection remains well within the small displacement assumption $\left(W_{\max }<h\right)$, and match very well within a relative discrepancy of $\pm 5 \%$ to the numerical results for all cases that consider the water-added mass.

Sensitivity to FSI parameter $\psi$. The peak deflections are now normalized by using Taylor's maximum displacement (no cavitation), see Eq. 28, and plotted as a function of the time ratio $\tau / T_{0}$ as well as the FSI parameter $\psi$ in Fig. 10. In fact, the only parameter varied here is the decay time $\tau$. However, it is believed that for the same stiffness, the relationship depicted in Fig. 10 would hold true for any combinations of peak pressures and decay times as long as the deformation remains in linear elastic domain. Decrease of the decay time or increase of the areal mass would result the decrease of the FSI parameter $\psi$, and vice versa. It is seen that the applicable domain is not the same between the thin and thick CFRP plates because varying the aspect ratio not only changes the lateral dimension (related to diffraction time, $T_{d}$ ) but also the plate stiffness (related to the swing time, $T_{0}$ ).

Relative error bar of $15 \%$ is shown in Fig. $10 \mathrm{a}$ and $10 \mathrm{~b}$ to evaluate the 
applicable domain of the proposed method. It can be seen that this range is $0.09 \leq \tau / T_{0} \leq 0.16(19.8 \leq \psi \leq 34.5)$ for thin plate and $0.28 \leq \tau / T_{0} \leq 0.35$ ${ }_{565}(4 \leq \psi \leq 5.1)$ for the thick plate. Any increase or decrease from those limiting values would result underestimation or overestimation of the normalized plate deflection compared to the results of LS-DYNA/USA acoustics simulations. The reason for the overestimation is mainly due to a prolong cavitation caused by the relatively low decay time of the loading or relatively large areal mass of the plate. Since water-added mass effect (or reloading) could only start at the end of the cavitation process, the consideration of the water-added mass from the beginning could yield overestimation in the proposed analytical formulations. On the other hand, when the time ratio $\tau / T_{0}$ or FSI parameter $\psi$ exceeds the upper bound of the proposed intervals, the analytical model underestimates the 575 plate deflection since the impulse-based nature of the current analytical model is unable to capture any continuing interaction between the fluid and structure, especially for relatively thick plates shown in Fig. 10b. In this case, cavitation may occur but it will either collapse very rapidly or will only occur at some point in the fluid away from the fluid-structure interface, either case suggesting that the transferred impulse given by Taylor's theory would be underestimated.

Effect of water-added mass. By considering the water-added mass in our analytical model, the Taylor's transferred impulse $I_{t}$ given by Eq. 21 is modified into:

$$
\begin{aligned}
I_{t_{\text {mod }}} & =I_{t_{\text {Taylor }}}+I_{t_{\text {water }}} \\
& =2 I_{0} \psi^{-\frac{\psi}{\psi-1}}+M_{a_{11}} v_{i} \\
& =2 I_{0} \psi^{-\frac{\psi}{\psi-1}}\left(1+\frac{M_{a_{11}}}{\rho h}\right)
\end{aligned}
$$

where the factor $\left(1+\frac{M_{a_{11}}}{\rho h}\right)$ represents the increase of the response due to wateradded mass. Note that only mode $[1,1]$ of the added mass has been considered in the above equation since its contribution to the plate response is by far the highest. Such effect, indeed, extends the limit of Taylor's theory to some extent (recall that using only Taylor's impulsive result would yield underestimation in 


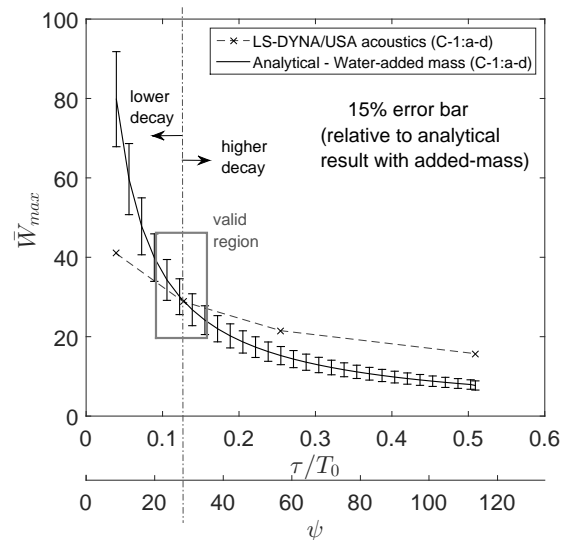

(a) Thin CFRP plate $(a / h=69.4)$

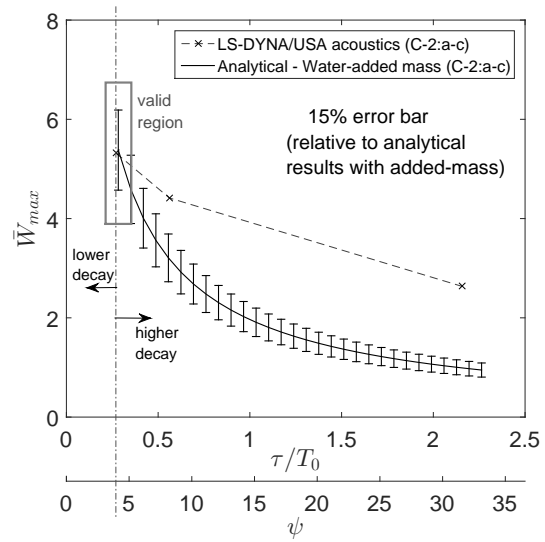

(b) Thick CFRP plate $(a / h=17.4)$

Figure 10: Comparison of dimensionless maximum central-deflection results between analytical and numerical methods for thin and thick rectangular CFRP plates

any cases). The amount of amplification is directly proportional to the lateral dimension of the plate, that is, the larger the plate, the more amplification of the response due to water-added mass will be obtained. Based on the present studies, relatively larger plates are more prone to cavitation since their swing times $T_{0}$ as well as diffraction time $T_{d}$ are usually longer compared to the decay times $\tau$. In addition, it will take much longer for the cavitation to collapse if $\tau_{c} \ll T_{d}$. As already discussed, the longer the cavitation phase, the more overestimation the current formulation will be resulted since the proposed method does not consider a time gap between the initiation of the cavitation and the reattachment of the water to the plate after cavitation collapses.

In Fig. 11, dimensionless transferred impulse with or without water-added mass as well as the dimensionless maximum impulsive velocity are plotted as a function of Taylor's FSI coefficients. As $\psi$ tends to infinity, both transferred impulse with or without water-added mass will approach to zero. The dimensionless maximum impulsive velocity will approach to 2. This is exactly analogous to the original free-rigid plate FSI theory of Taylor [10] except that the range of applicability is extended by increasing $\bar{I}_{t}$ or equivalently by decreasing $\psi$ of 
Taylor by an amount $\psi_{\text {water }}=\left(\tau \rho_{w} c_{w}\right) /\left(\rho h+M_{a_{11}}\right)$. Note that the impulsive velocity $\bar{v}_{i}$ will be the same for both analytical models since the increase in momentum $I_{t_{\text {water }}}$ and increase in mass $M_{a_{11}}$ will cancel each other out. A more versatile approach that includes not only the effect of cavitation but also the continuing FSI effect related to relatively long decay times should be considered in the future.

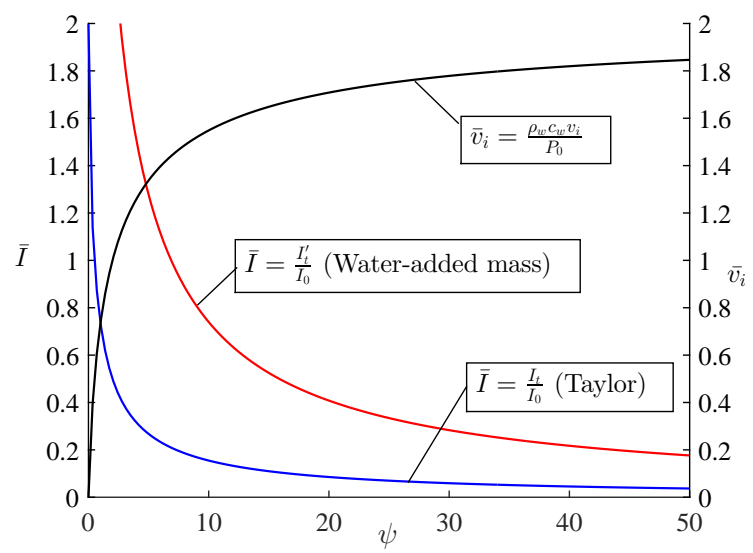

Figure 11: Dimensionless transferred impulse and impulsive velocity as a function of Taylor's FSI coefficient (Calculation is done based on thick CFRP plates with $h / a=17.4$ )

Sensitivity to plate stiffness. Now the effect of changing the plate stiffness will be investigated by keeping the same areal mass and the same decay time so as to have the same FSI parameter $\psi$. Comparisons will be made for long lateral dimension $(a=b=0.4 \mathrm{~m})$ and for short lateral dimension $(a=b=0.1 \mathrm{~m})$. It should be kept in mind that the lateral dimension is associated to the diffraction time and thus, keeping the same lateral dimension will give the same diffraction time $T_{d}$ and similarly, the same FSI parameter $\psi$ and decay time $\tau$ will yield the same cavitation inception time $\tau_{c}$ according to Eq. 20. Therefore, the effect of change will be solely due to the plate areal stiffness $K$. Peak pressures are adjusted in order to keep the analysis well within the linear elastic, small displacement domain, that is, $W_{\max }<h$. FSI parameter of $\psi=28.8$ and $\psi=4.14$ are selected for long plate $(a=0.4 \mathrm{~m})$ and short plate $(a=0.1 \mathrm{~m})$ 
respectively. The objective here is to verify whether the applicable range (within the relative error margin of $\pm 15 \%$ ) specified in the previous discussions is still valid for any changes in the stiffness assuming a negligible structural damping. The following ply orientations are considered:

- layout 1: $[ \pm 45 / 0 / 0 / 0 / \pm 45 / 0 / 0 / 0 / 90 / 90]_{S}$ — with 20 plies,

- layout 2: $[0 / 90 / 0 / 90 / \ldots / 0 / 90 / 0 / 90]$ — with 20 plies.

To investigate the change of stiffness due to material, carbon-fiber/epoxy (CFRP) and glass-fiber/epoxy (GRP) plates are employed. The corresponding properties, extracted from quasi-static tests done by the authors, are given in Table 6 and 8 for CFRP and GRP respectively.

The results for both long and short lateral dimensions are shown in Fig. 12. The corresponding FSI parameter $\psi$, aspect ratios $a / h$, material, ply layout and total areal stiffness $K$ (see Eq. 32 ) are also given in each figure. Note that the thickness of the GRP plate needs to be adjusted in order to have the same areal mass. First of all, it can be said that the applicable domain agrees well with the previously deduced values, $0.09 \leq \tau / T_{0} \leq 0.16(19.8 \leq \psi \leq 34.5)$ for relatively thin plate and $0.28 \leq \tau / T_{0} \leq 0.35(4 \leq \psi \leq 5.1)$ for relatively thick plate. The relative error range is also well within the acceptable values, $15 \%$ and $5 \%$ for relatively long and short plates respectively. In addition to this, the pattern observed is also in consistence with the expected behavior, that is, when the plate becomes more flexible (as $\tau / T_{0}$ gets smaller), cavitation is more likely to occur and will last longer unlike the stiffer plates. Indeed, the rapid collapse of cavitation for the stiffer plates (i.e., short lateral dimensions) has made the

Table 8: Characteristics of the material (glass-fiber/epoxy laminate)

\begin{tabular}{cccccc}
\hline $\begin{array}{c}E_{11} \\
(\mathrm{GPa})\end{array}$ & $\begin{array}{c}E_{22}=E_{33} \\
(\mathrm{GPa})\end{array}$ & $\begin{array}{c}\nu_{12}=\nu_{13} \\
-\end{array}$ & $\begin{array}{c}\nu_{23} \\
G_{12}=G_{13} \\
(\mathrm{GPa})\end{array}$ & $\begin{array}{c}G_{23} \\
(\mathrm{GPa})\end{array}$ \\
\hline 34.1 & 10 & 0.279 & 0.402 & 3.03 & 3.58 \\
\hline
\end{tabular}




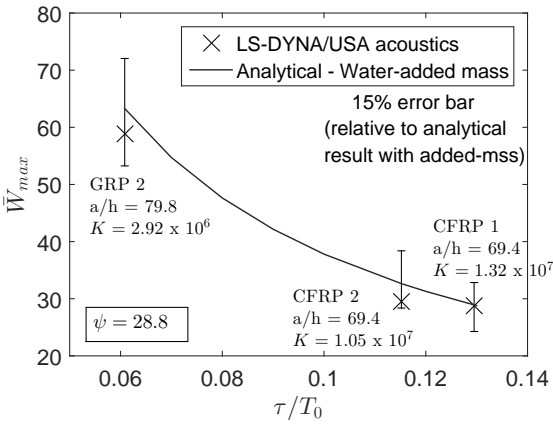

(a) Long lateral dimensions $(a=b=0.4 \mathrm{~m})$

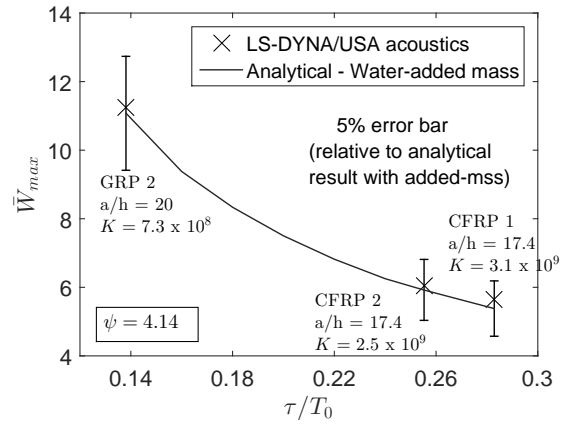

(b) Short lateral dimensions $(a=b=0.1 \mathrm{~m})$

Figure 12: Effect of stiffness for carbon-fiber/epoxy and glass-fiber/epoxy plates with different stacking sequences, layout 1 and 2 (denoted by 'CFRP1', 'CFRP2' and 'GRP2' respectively)

comparison with the analytical two-step impulse-based approach to be much more relevant since the proposed method considers an abrupt transfer of energy due to water-added mass without any time gap between cavitation appearance and water reattachment.

\section{Conclusions}

Several conclusions as well as important observations can be made from this research work. The performances of different numerical approaches to capture the interactions between a plate subjected to the shock wave of an underwater explosion and the surrounding medium are evaluated in details and compared to the experimental results both from the literature and an in-house developed experimental test data from DGA. According to the observations based on the case studies adapted in this paper, it was found out that LS-DYNA/USA acoustic model shows the best correlations with the experiment. Using only impulsive velocity given by Taylor could lead to significant underestimations especially for thick plates, which oscillate in high frequencies. Using only DAA approach (i.e without modeling explicitly the surrounding acoustic fluid) could overestimate the responses especially for relatively large and thin plates in which cavitation is more likely to occur and could last longer depending on the duration of the 
incident shock wave. Using LS-DYNA/USA (only acoustics) simulations with only finite extent of water may lead to underestimations and an unnaturally slow rebounding of the plate due to confined pressures in the neighboring acoustic volume elements.

Indeed, these numerical codes are very powerful and have been successively employed over the past decades but the complexity as well as the time needed to perform the computations should be aware. Even with the most powerful code, LS-DYNA/USA acoustics, there can be some problems such as numerical instability and many artificial numerical terms may need to be handled carefully. Moreover, owing to the involvement of large water domain and the rigid baffle plate, numerical simulations can be very time-consuming especially for relatively large composite plates studied in this paper. From the industrial point of view, it is not practical to use such expensive tools to perform preliminary design studies that may involve a large number of configurations. That is why simplified analytical solutions are proposed by adapting a twostep approach based on transferred impulse provided by Taylor's theory for the early time and water-added mass for the long time response. The focus has been paid for the application of this method on the UNDEX response of airbacked composite rectangular plates, emphasizing more on the FSI effect within linear-small displacement domain. Different parametric studies such as varying the peak pressures, decay times, aspect ratios, areal mass as well as stiffness corresponding to different materials and ply orientations are performed and a number of important phenomena are observed. Finally, the applicable limit of the two-step approach is exposed in terms of the time ratio between the decay time of the loading and the plate in-air swing time as well as in terms of the FSI parameter that relates decay time and areal mass. It was found out that within this applicable region, varying of either the materials, stacking sequences of the laminate or the peak pressures would still allow to estimate the response within a relative error of $\pm 15 \%$ according to some of the studies performed in this research work.

It must, however, be pointed out that the simplified two-step approach is 
valid only for the limiting ranges pointed out for corresponding aspect ratio.

Indeed, many of the other phenomena such as the non-linearity due to large deformation, the propagation of breaking and closing fronts caused by cavitation, the continuing FSI effect due to relatively long shock loading, effect of hydrostatic pressure, change in the backed condition such as water and the effect of damage should be studied in the future.

\section{Acknowledgements}

This research work has been conducted with the financial grant of DGADGE. The authors would also like to express their gratitude to DGA for providing the experimental data, Calcul-Meca and Multiplast companies for their technical support.

\section{Appendix}

The mass terms $M_{1}, M_{2}$ and $M_{3}$ are as follows:

$$
\begin{aligned}
& M_{1}=\rho_{c} \sum_{k=1}^{N} h_{k}-h_{k-1} \\
& M_{2}=M_{3} \approx 0
\end{aligned}
$$

The stiffness terms $K_{i j_{m n}}(i, j=1,2,3)$ are as follows:

$$
\begin{aligned}
& K_{11}=A_{44}\left(\frac{n \pi}{b}\right)^{2}+A_{55}\left(\frac{m \pi}{a}\right)^{2} \\
& K_{12}=A_{55}\left(\frac{m \pi}{a}\right) \\
& K_{13}=A_{44}\left(\frac{n \pi}{b}\right) \\
& K_{22}=D_{11}\left(\frac{m \pi}{a}\right)^{2}+D_{66}\left(\frac{n \pi}{b}\right)^{2}+A_{55} \\
& K_{23}=\frac{m n \pi^{2}}{a b}\left(D_{11}+D_{66}\right) \\
& K_{33}=D_{22}\left(\frac{n \pi}{b}\right)^{2}+D_{66}\left(\frac{m \pi}{a}\right)^{2}+A_{44}
\end{aligned}
$$

The overall areal stiffness of the plate for mode $(m, n)$ is:

$$
K_{m n}=K_{11}+\frac{2 K_{12} K_{23} K_{13}-\left(K_{12}^{2} K_{33}+K_{13}^{2} K_{22}\right)}{K_{22} K_{33}-K_{23}^{2}}
$$




\section{References}

[1] R. H. Cole, Underwater explosions, Princeton University Press, Princeton, 1948.

[2] H. U. Mair, Benchmarks for submerged structure response to underwater explosions, Shock and Vibration 6 (4) (1999) 169-181. doi:10.1155/1999/ 743708 .

[3] M. Porfiri, N. Gupta, A review of research on impulsive loading of marine composites, Major Accomplishments in Composite Materials and Sandwich Structures: An Anthology of ONR Sponsored Research (2009) 169-194.

[4] A. H. Keil, The Response of Ships to Underwater Explosions, in: Annual Meeting, The Society of Naval Architects and Marine Engineers, New York, N.Y., 1961, pp. 366-410.

[5] D. J. Hall, Examination of the effects of underwater blasts on sandwich composite structures, Composite Structures 11 (2) (1989) 101-120.

[6] A. P. Mouritz, The effect of underwater explosion shock loading on the flexural properties of GRP laminates, International Journal of Impact Engineering 18 (2) (1996) 129-139.

[7] J. Leblanc, A. Shukla, Dynamic response and damage evolution in composite materials subjected to underwater explosive loading: An experimental and computational study, Composite Structures 92 (10) (2010) 2421-2430. doi:10.1016/j.compstruct.2010.02.017.

[8] V. Deshpande, A. Heaver, N. Fleck, An underwater shock simulator, in: Proceedings of the Royal Society A, no. 462, Cambridge, UK, 2006, pp. 1021-1041. doi:10.1098/rspa.2005.1604

${ }_{730}$ [9] A. Schiffer, V. L. Tagarielli, The response of circular composite plates to underwater blast: Experiments and modelling, Journal of Fluids and Structures 52 (2015) 130-144. doi:10.1016/j.jfluidstructs.2014.10.009. 
[10] G. Taylor, The pressure and impulse of submarine explosion waves on plates, in: The Scientific Papers of G. I. Taylor, Vol. III, Cambridge University Press, Cambridge, UK, 1941, pp. 287-303.

[11] E. Kennard, Cavitation in an Elastic Liquid, Physical Review 63 (5 and 6) (1943) 172-181.

[12] E. Kennard, The effect of a pressure wave on a plate or diaphragm, Tech. rep., Navy Department, David Taylor Model Basin, Washington, D.C. (1944).

[13] A. Schiffer, V. Tagarielli, N. Petrinic, A. Cocks, The Response of Rigid Plates to Deep Water Blast : Analytical Models and Finite Element Predictions, Journal of Applied Mechanics 79 (2012). doi:10.1115/1.4006458.

[14] H. H. Bleich, I. S. Sandler, Interaction between structures and bilinear fluids, International Journal of Solids and Structures 6 (1970) 617-639. doi: 10.1016/0020-7683(70)90034-X.

[15] C. Felippa, J. DeRuntz, Finite Element Analysis of Shock-induced Hull Cavitation, Computer Methods in Applied Mechanics and Engineering 44 (1984) 297-337.

[16] T. L. Geers, Residual potential and approximate methods for three dimensional fluid-structure interaction problems, The Journal of the Acoustical Society of America 49 (1971) 1505-1510.

[17] T. L. Geers, Doubly asymptotic approximations for transient motions of submerged structures, The Journal of the Acoustical Society of America 64 (1978) 1500-1508.

[18] J. A. DeRuntz Jr., The underwater shock analysis code and its applications, in: Proceedings of the 60th Shock and Vibration Symposium, 1989, pp. 89107. 
[19] R. Newton, Effects of cavitation on underwater shock loading - Part I, Tech. rep., Naval Post-graduate School, Monterey, CA. (1978).

URL http://hdl.handle.net/10945/29265

[20] G. Barras, Fluid-structure interaction: application to close-in underwater explosion., Ph.d thesis, University of Sciences and Technologies, Lille, France. (2012).

[21] K. Brochard, H. Le Sourne, G. Barras, Extension of the string-onfoundation method to study the shock wave response of an immersed cylinder, International Journal of Impact Engineering 117 (May 2017) (2018) 138-152. doi:10.1016/j.ijimpeng.2018.03.007.

[22] Z. Xue, J. W. Hutchinson, A comparative study of impulse-resistant metal sandwich plates, International Journal of Impact Engineering 30 (10) (2004) 1283-1305. doi:10.1016/j.ijimpeng.2003.08.007.

[23] Y. Liang, A. V. Spuskanyuk, S. E. Flores, D. R. Hayhurst, J. W. Hutchinson, R. M. McMeeking, A. G. Evans, The response of metallic sandwich panels to water blast, Journal of Applied Mechanics, Transactions ASME 74 (1) (2007) 81-99. doi:10.1115/1.2178837.

[24] A. Schiffer, V. L. Tagarielli, The dynamic response of composite plates to underwater blast: Theoretical and numerical modelling, International

n Journal of Impact Engineering 70 (2014) 1-13. doi:10.1016/j.ijimpeng. 2014.03 .002

[25] M. S. Hoo Fatt, D. Sirivolu, Marine composite sandwich plates under air and water blasts, Marine Structures 56 (2017) 163-185. doi:10.1016/j. marstruc.2017.08.004

[26] J. Reddy, Mechanics of Laminated Composite Plates and Shells, 2nd Edition, CRC Press LLC, Florida, 2004. 
[27] M. Hoo Fatt, L. Palla, Analytical modeling of composite sandwich panels under blast loads, Journal of Sandwich Structures and Materials 11 (4) (2009) 357-380. doi:10.1177/1099636209104515

[28] J. E. Greenspon, Vibrations of Cross-stiffened and Sandwich Plates with Application to Underwater Sound Radiators, The Journal of the Acoustical Society of America 33 (11) (1961) 1485-1497.

[29] LSTC, LS-DYNA: Keyword User's Manual, Vol. 1, Livermore Software Technology Corporation, California, 2018.

URL https://www . dynasupport.com/manuals

[30] T. L. Geers, K. S. Hunter, An integrated wave-effects model for an underwater explosion bubble, The Journal of the Acoustical Society of America 111 (4) (2002) 1584-1601. doi:10.1121/1.1458590.

[31] C. A. Felippa, A Family of Early-Time Approximations for Fluid-Structure Interaction, Journal of Applied Mechanics 47 (1980) 703-708.

[32] J. N. Reddy, Geometrically Nonlinear Transient Analysis of Laminated 